\title{
Assisted Human-Robot-Interaction for Industrial Assembly
}

\author{
Application of Spatial Augmented Reality (SAR) for Collaborative Assembly Tasks
}

\author{
Jan Schmitt ${ }^{\dagger}$ \\ University of Applied \\ Sciences, Würzburg- \\ Schweinfurt, Germany \\ Institute Digital Engineering \\ jan.schmitt@fhws.de
}

\author{
Philipp Kranz \\ University of Applied \\ Sciences, Würzburg- \\ Schweinfurt, Germany \\ Institute Digital Engineering
}

\author{
Tobias Kaupp \\ University of Applied \\ Sciences, Würzburg- \\ Schweinfurt, Germany \\ Institute Digital Engineering \\ tobias.kaupp@fhws.de
}

\begin{abstract}
Human-robot collaboration is increasingly applied to industrial assembly sequences due to the growing need for flexibility in manufacturing. Assistant systems are able help to support shared assembly sequences to facilitate collaboration. This contribution shows a workplace installation of a collaborative robot (Cobot) and a spatial augmented reality (SAR) assistant system applied to an assembly use-case. We demonstrate a methodology for the distribution of the assembly sequence between the worker, the Cobot, and the SAR.
\end{abstract}

KEYWORDS: Industrial assembly, Worker assistance, Cobot, Spatial Augmented Reality

\section{ACM Reference format:}

Jan Schmitt, Andreas Hillenbrand, Philipp Kranz and Tobias Kaupp. 2021. Assisted Human-Robot-Interaction in Industrial Assembly: Application of Spatial Augmented Reality in Collaborative Assembly Tasks. In Companion of the 2021 ACM/IEEE International Conference on Human-Robot Interaction (HRI'21 Companion), March 8-11, 2021, Boulder, CO, USA. ACM, New York, NY, USA, 5 pages. https://doi.org/10.1145/3434074.3447127

\section{Introduction}

Due to the recent trend of customer-specific mass production, industrial assembly is exposed to increasing competitive pressure. As a result, companies face challenges of economic and technological optimization. Modern production systems must be flexible to adapt to increasing product complexity and at the same time handle the increasing number of variants, decreasing production lot sizes, as well as shorter product life cycles. A high product quality is to be maintained while at the same time, costs need to be kept at a minimum. To overcome those production challenges, collaborative robots (Cobots) find their way into industrial applications to enable human-robot interaction (HRI)

${ }^{\dagger}$ Corresponding author

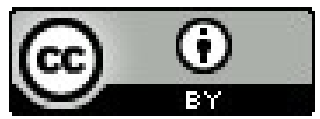

This work is licensed under a Creative Commons Attribution International 4.0 License.

HRI '21 Companion, March 8-11, 2021, Boulder, CO, USA.

(C) 2021 Copyright held by the owner/author(s).

ACM ISBN 978-1-4503-8290-8/21/03. https://doi.org/10.1145/3434074.3447127 with different characteristics of a collaboration. On the one hand, Cobots are flexible and enable semi-automated production systems as they can be operated without safety fences next to human workers. On the other hand, the strengths of each party can be combined, e.g. the human's sensory abilities and the robot's repeatability, and ergonomics are improved. Nevertheless, it must be noted that the broad use of Cobots depends on further aspects. Worker acceptance, a reasonable and clear assignment of work contents, as well as interaction and documentation mechanisms for the shared assembly sequences are crucial factors for the adoption of of Cobots in industry [1]. In this contribution, we demonstrate our approach of assisted HRI in industrial assembly by utilizing spatial augmented reality (SAR) as follows:

- Display of dedicated work areas, e.g. worker, robot, collaborative

- Smart visualization of worker-centered information

- Control of the assembly sequence via virtual buttons

The combination of a Cobot, a spatial augmented reality system, and a human worker may enhance user acceptance due to the adaptable visible information provided during the assembly [2]. Following this approach, we developed a use-case for the (interactive) assembly of a toy truck via a team consisting of a worker, a Cobot, and a projection system. We integrated the interaction mechanisms of coexistence, interaction, cooperation, and collaboration (according to the definition of Wang et al. [3]) and extended those by spatial augmentation.

Section 2 briefly reviews existing research approaches of HRI in industrial assembly and the utilization of worker assistance systems. Subsequently, our assisted workstation is presented. Section 3 introduces the use case including assembly tasks, how they have been distributed between the Cobot and the worker and what kind of assistive elements have been realized.

\section{Related Work}

HRI in manufacturing or assembly environments is already widespread [4]. The Cobot-inherent safety features e.g. forcetorque control enable a safe collaboration between the robot and the worker. Considering ergonomics and user acceptance, attention must be paid to an adapted motion sequence at the appropriate speed to avoid any additional strain on the person [5, 6]. In terms of production efficiency, Cobots can significantly increase productivity and flexibility of the assembly process due to the parallelization of manual and automated work sequences. Prior 
research has investigated the effects of assistance systems on the productivity of assembly tasks, e.g. by providing additional information or technical documentation via AR. Comparing ARbased assistance systems with conventional printed instructions, it could be shown that a projection on the work desktop and on the object provides better support than other technologies [7]. A reduction in cognitive load, lead time, and error rate can be observed while flexibility in assembly increases. Furthermore, the limits and possibilities of different AR-assistance systems have been investigated and recommendations for action as well as design-for-learning and health promotion have been developed [8]. A general acceptance of AR-based assistance systems has been determined in user studies, e.g. by [9]. Workstations consisting of a combination of an assistance system with a Cobot are rarely discussed in literature. Wang et al. [3] examined the potential of introducing AR technologies in a worker assistance system for HRI. Here, robot commands and work instructions can be extended virtually for the HRI. The overall architecture of the ARbased worker support system was designed and a test case was created. Blaga and Tamas [10] combine a Cobot with data glasses at a workstation. Their goal is to perform cooperative and collaborative tasks in industrial assembly. They additionally use the 3D model of the workpiece to be processed and display it in the field of view of the worker using a HoloLens. The aim of the work of Makris et al. [11] was the development of a system that displays assembly instructions in the worker's field of view when working with a Cobot. For this purpose, they combined a Cobot with data glasses and a smartwatch. The system was designed to increase user safety and acceptance for working near large industrial robots. Charoenseang and Tonggoed [12] combined a camera system with a Cobot for hybrid assembly activities. Using AR technology, objects are superimposed on a live image of the workstation on a monitor to assist in the assembly process. Results showed

1. a reduction in the assembly time, and

2. that graphical instructions better support the worker in their interactions with Cobos when compared to text.

The motivation of Ender and Wagner et al. [13] was to reduce the physical and cognitive stress on users during assembly tasks and thus increase productivity. The results described in this research show an optimization of workplaces for manual production and maintenance processes. A hybrid workstation consisting of a Cobot, a camera system and a projector was built in a digital learning factory. The gestures of the worker are followed by the camera system and interpreted and evaluated by a control computer. Based on the learned behavior, the robot sequences are generated automatically. If a captured gesture of the operator is new, the data are transmitted to an artificial intelligence model and the behavior strategy of the system is adopted. Only further investigations will show whether the developed approach delivers the desired results. The comprehensive results of the literature review are shown in Table 1 . The research papers are categorized according to the system elements of an assistive workstation Cobot, AR assistant system and their combination.
Table 1. Categorized Literature Review

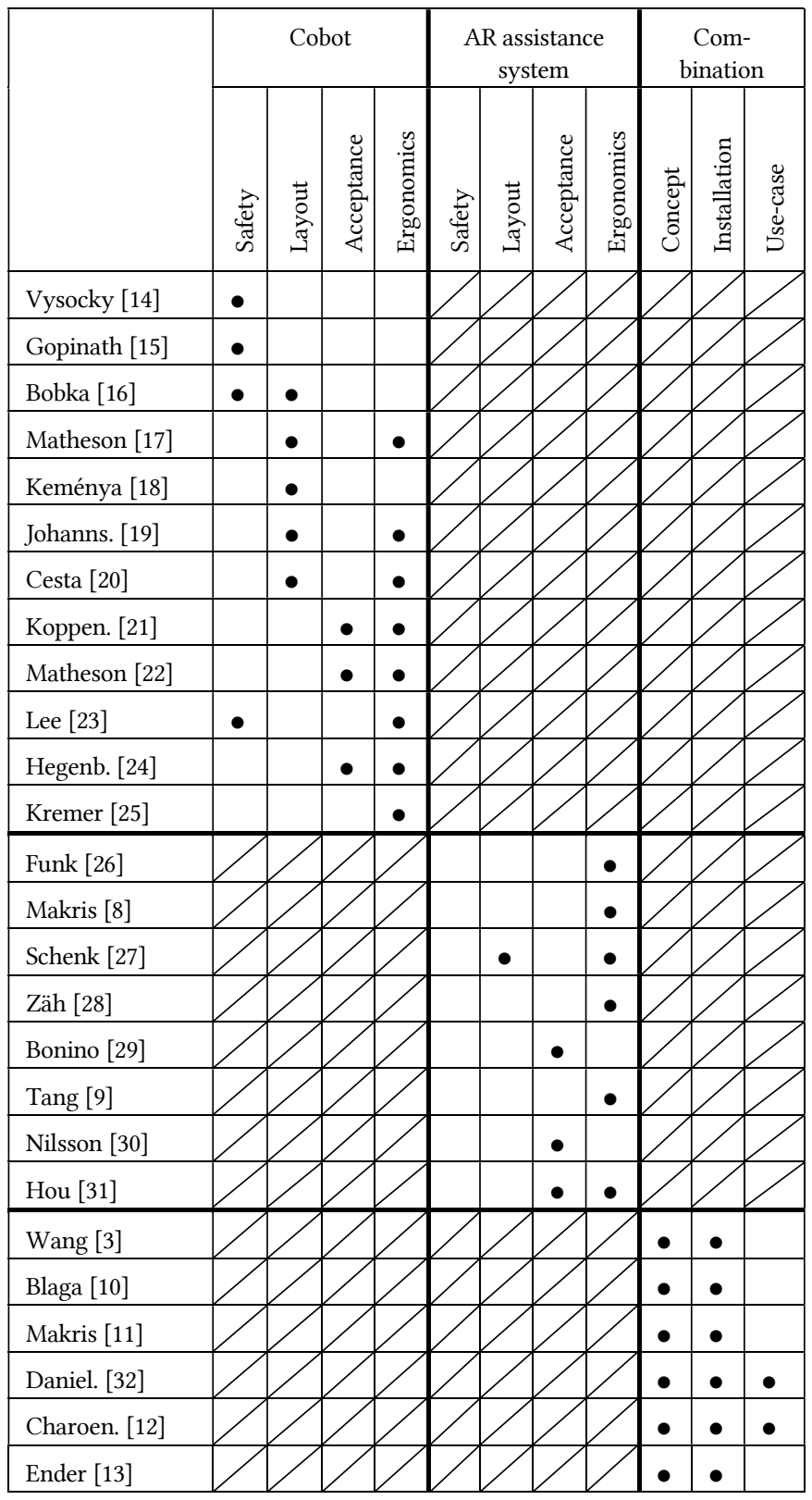

The following can be derived from Table 1 :

1. For Cobots, user acceptance research is rarely mentioned in literature

2. For AR assistance systems, safety and layout issues are not central research topics

3. The combination of Cobots and assistance systems is rarely demonstrated on actual use cases relevant for industrial assembly scenarios.

In our work, we realized an assisted HRI workplace for industrial assembly tasks capable of incorporating various assistive HRI sequences and demonstrate those in a use case. The next section shows the conceptual approach as well as the installed industrylike workplace. 


\section{Assisted Human-Robot Workplace}

The motivation of assisted HRI workplaces for industrial assembly tasks is the combination of two promising approaches: HRI and assisted/smart workstations. The concept of collaboration between humans and robots is intended to optimally combine the competencies of both - sensory or tactile abilities of the human supported by the precision, repeat accuracy or power of a robot. Furthermore, workers can be supported by assistance systems while carrying out their tasks. Assistance systems promise to improve the digital process chain and to increase productivity even for more complex assembly tasks. A reduced assembly error rate can be achieved by controlling the work steps explicitly and taking in-process measurements. Assistance systems also facilitate the training of employees. Figure 1 shows the workplace we created for our research: it is comprised of a Cobot (Han's Elfin 5) and a projector/camera/depth camera system (Ulixes A600), mounted on a height-adjustable workbench with small parts storage and electrical torque screwdrivers.

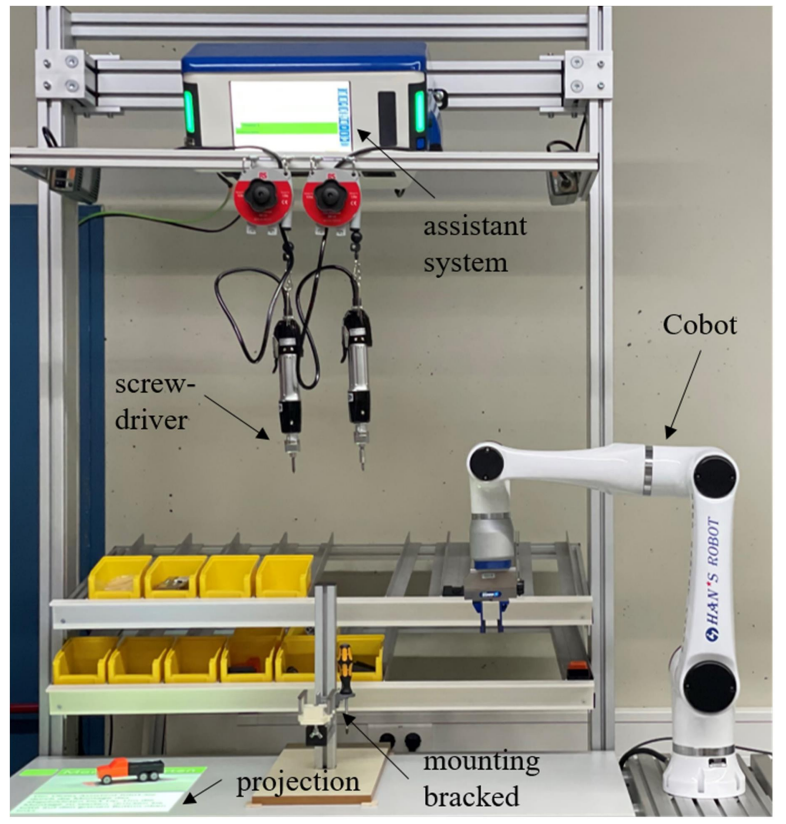

Figure 1: Assisted HRI workplace

To ensure a continuous flow of information, the elements of the workplace are interconnected as follows: the Cobot and the assistance system are connected via digital I/Os (24V. The communication and interaction with the worker is realized via visual information projected on the desktop. Those visual assets are

- virtual buttons

- assembly instructions (text, picture, video)

- markers indicating the assembly position

- area borders to indicate dedicated workspaces

A direct communication between the Cobot and the worker has not been established. To evaluate the concept for industrial assembly, a use-case demonstrates the assistive HRI mechanisms as discussed next.

\section{Use-Case: Toy Truck Assembly}

The use-case consists of a toy truck assembly with 17 parts. The truck is manufactured in an Industry 4.0 pilot factory located at our University. The truck can be configured in different variants - long/short chassis, with/without a double-axle in the back (see Figure 2).

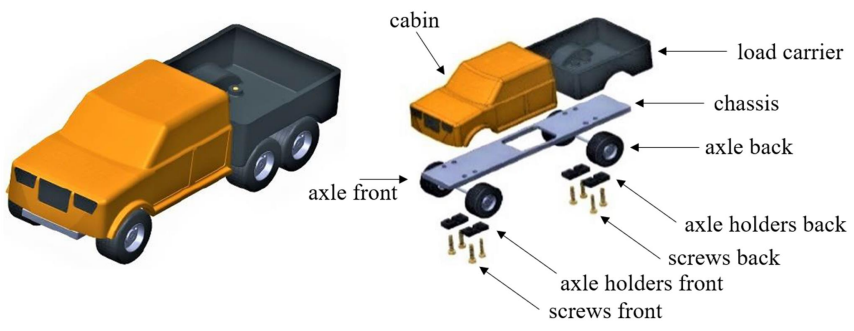

Figure 2: 3D model of the toy truck, variant with three axles (left), 3D exploded view of the toy truck with two axles (right)

The parts are assembled according to the priority matrix shown in Table 2. For simplification purposes, the axle holders and screws are considered as one element for front and back of the truck.

Table 2: Assembly priority matrix

\begin{tabular}{|c|c|c|c|c|c|c|c|c|c|}
\hline & $\begin{array}{l}\text {.ี } \\
\text { त्ष } \\
\end{array}$ & 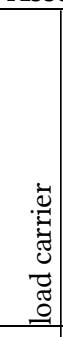 & 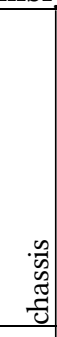 & 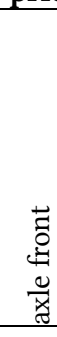 & 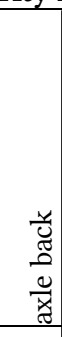 & 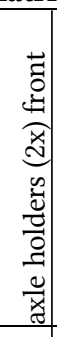 & 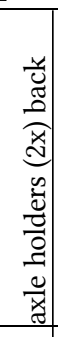 & 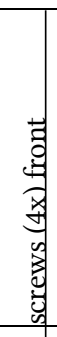 & 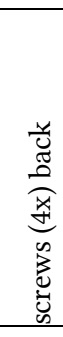 \\
\hline cabin & - & -1 & 1 & 1 & 1 & 1 & 1 & 1 & 1 \\
\hline load carrier & 1 & - & 1 & 1 & 1 & 1 & 1 & 1 & 1 \\
\hline chassis & -1 & -1 & - & 1 & 1 & 1 & 1 & 1 & 1 \\
\hline axle front & -1 & -1 & -1 & - & 0 & 1 & 1 & 1 & 1 \\
\hline axle back & -1 & -1 & -1 & 0 & - & 1 & 1 & 1 & 1 \\
\hline ax. hol. front & -1 & -1 & -1 & -1 & -1 & - & 0 & 1 & 0 \\
\hline ax. hol. back & -1 & -1 & -1 & -1 & -1 & 0 & - & 0 & 1 \\
\hline screws front & -1 & -1 & -1 & -1 & -1 & 1 & 0 & - & 0 \\
\hline screws back & -1 & -1 & -1 & -1 & -1 & 1 & -1 & 0 & - \\
\hline $\begin{array}{r}-1 \\
0 \\
1\end{array}$ & \multicolumn{9}{|c|}{$\begin{array}{l}\text { assembly of part } \mathrm{X} \text { after part } \mathrm{Y} \\
\text { assembly order is arbitrary } \\
\text { assembly of part } \mathrm{X} \text { before part } \mathrm{Y}\end{array}$} \\
\hline
\end{tabular}

The axle plate is placed on the cabin and the load carrier, both are lying upside down in a mounting bracket. The axles and the axle holder are placed and then screwed together.

The use of an assistance system means that the assembly sequences the worker and/or the robot conduct can be visually supported. The sequences must be designed according to the assembly priority while utilizing the strengths of humans and robots. Figure 3 illustrates the realized use-case as a flowchart. The 
assistance system is used as a master controller and coordinates the workflow between worker and robot. The camera of the assistance system records the activation of virtual buttons. Information for the worker is provided via projections on the desktop. The assistance system and the Cobot interact via their 24 V I/O control signals.

\begin{tabular}{|l|l|l|}
\hline \multicolumn{1}{|c|}{ Assistant system } & \multicolumn{1}{|c|}{ Cobot } \\
\hline start screen projection, virtual \\
button to start the sequence
\end{tabular}

Figure 3: Flow chart of the working sequences

Figure 4 shows three human-robot interaction types, each of which is supported by the AR system:

1. Coexistence: the robot picks up the load carrier, cabin, chassis and axles from their storage bins while the worker prepares the axle carriers and screws. The AR system projects the two separate work areas onto the workbench facilitating situational awareness for the worker.

2. Cooperation: the assistance system checks the correct position of the parts using its camera. The worker acknowledges the execution of the task

3. Collaboration: after inserting the first axle, the robot holds it in place while the worker mounts the axle holders using the screws. Therefore, the robot acts as a "third hand" to the worker here. The assistive system conducts a quality control step by checking that all screws have been inserted using its built-in camera.

At the end, a virtual button to complete the assembly step is projected on the work bench. For the assembly of further axles, this process is repeated.

Figure 4 shows examples for the visual cues the SAR elements provide during assembly. The use-case contains several assistive elements, where a SAR helps the worker to fulfill the assembly tasks, has to confirm the assembly operation or can watch where the main workspaces of himself/herself, the Cobot and the shared workspace is. Further, we utilize the Cobot as a flexible part fixing device ("third hand") for the axle while the worker is screwing.
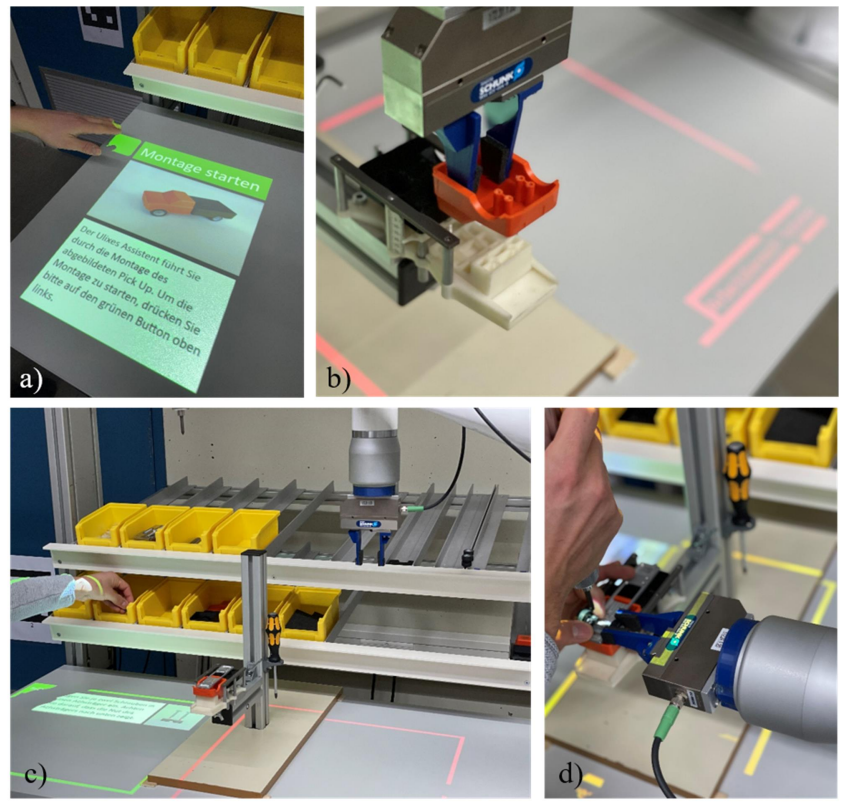

Figure 4: Assisted HRI elements - a) virtual button and assembly instructions, b) Cobot handles cabin in the area marked in red, c) parallel sequence with areas marked in green (worker) and red (Cobot) and d) collaborative sequence with Cobot as " 3 rd hand" marked in yellow.

\section{Conclusion and Further Research}

Based on the motivation to make manual assembly tasks more productive while ensuring that workers accept "colleague Cobot", we present an SAR-assisted workplace. We demonstrated three types of interactions by applying the system to the use-case of a toy truck assembly. We presented how we split the working sequence according to competencies and showed the SAR- and Cobot-assisted elements that were used. Further research is planned as follows:

1. Implementation of a use case from our industry partner to verify the generalizability and applicability of our approach

2. Verification of increased productivity and user acceptance compared to manual assembly processes

3. Development and publication of an industry guideline for the systematic creation of assisted HRI workplaces for manual assembly tasks.

\section{ACKNOWLEDGMENTS}

The authors gratefully acknowledge the support by the state of Bavaria (Germany). The Bayerische Forschungsstiftung funds this research project InKoMo under the grant no. AZ-1401-19.

\section{REFERENCES}

[1] Gattullo, M., Dammacco, L., Francesca Ruospo, F., Evangelista, A., Fiorentino, M., Schmitt, J., Uva, A.-E. (2020). Design preferences on Industrial Augmented Reality: a survey with potential technical writers. IEEE International Symposium on Mixed and Augmented Reality (ISMAR), Nov. 2020 
[2] Schuster, F., Sponholz, U., Engelmann, B., Schmitt, J. (2020). A User Study on ARassisted Industrial Assembly. IEEE International Symposium on Mixed and Augmented Reality (ISMAR), Nov. 2020

[3] Wang, L., Gao, R., Váncza, J., Krüger, J., Wang, X. V., Makris, S., \& Chryssolouris, G. (2019). Symbiotic human-robot collaborative assembly. CIRP annals, 68(2), 701-726.

[4] Huber, A., \& Weiss, A. (2017, March). Developing human-robot interaction for an industry 4.0 robot: How industry workers helped to improve remote-HRI to physical-HRI. In Proceedings of the Companion of the 2017 ACM/IEEE International Conference on Human-Robot Interaction (pp. 137-138).

[5] Hegenberg, J., Schimpf, D. W., Fischer, N., \& Schmidt, L. (2018). Fallstudie zur Roboterunterstützung des Menschen bei manueller Montage. Zeitschrift für Arbeitswissenschaft, 72(4), 239-251.

[6] Kremer, D., \& Hermann, S. Arbeitsplätze für die Mensch-Roboter-Kollaboration inklusionsförderlich und wirtschaftlich gestalten.

[7] Funk, M., Kosch, T., \& Schmidt, A. (2016, September). Interactive worker assistance: comparing the effects of in-situ projection, head-mounted displays, tablet, and paper instructions. In Proceedings of the 2016 ACM International Joint Conference on Pervasive and Ubiquitous Computing (pp. 934-939).

[8] Makris, S., Pintzos, G., Rentzos, L., \& Chryssolouris, G. (2013). Assembly support using AR technology based on automatic sequence generation. CIRP Annals, 62(1), 9-12.

[9] Tang, A., Owen, C., Biocca, F., \& Mou, W. (2003, April). Comparative effectiveness of augmented reality in object assembly. In Proceedings of the SIGCHI conference on Human factors in computing systems (pp. 73-80).

[10] Blaga, A., \& Tamas, L. (2018, June). Augmented reality for digital manufacturing. In 2018 26th Mediterranean Conference on Control and Automation (MED) (pp. 173-178). IEEE.

[11] Makris, S., Karagiannis, P., Koukas, S., \& Matthaiakis, A. S. (2016). Augmented reality system for operator support in human-robot collaborative assembly. CIRP Annals, 65(1), 61-64.

[12] Charoenseang, S., \& Tonggoed, T. (2011, July). Human-robot collaboration with augmented reality. In International Conference on Human-Computer Interaction (pp. 93-97). Springer, Berlin, Heidelberg.

[13] Ender, J., Wagner, J. C., Kunert, G., Guo, F. B., Larek, R., \& Pawletta, T. (2019) Concept of a self-learning workplace cell for worker assistance while collaboration with a robot within the self-adapting-production-planning-system Informatyka, Automatyka, Pomiary W Gospodarce I Ochronie Środowiska,, 9(4), 4-9.

[14] Vysocky, A. L. E. S., \& Novak, P. E. T. R. (2016). Human-Robot collaboration in industry. MM Science Journal, 9(2), 903-906.

[15] Gopinath, V., Ore, F., Grahn, S., \& Johansen, K. (2018). Safety-focussed design of collaborative assembly station with large industrial robots. Procedia manufacturing, 25, 503-510.

[16] Bobka, P., Germann, T., Heyn, J. K., Gerbers, R., Dietrich, F., \& Dröder, K. (2016) Simulation platform to investigate safe operation of human-robot collaboration systems. Procedia CIRP, 44, 187-192.

[17] Matheson, E., Minto, R., Zampieri, E. G., Faccio, M., \& Rosati, G. (2019). HumanRobot Collaboration in Manufacturing Applications: A Review. Robotics, 8(4), 100 .
[18] Kemény, Z., Beregi, R., Nacsa, J., Kardos, C., \& Horváth, D. (2018). Human-robot collaboration in the MTA SZTAKI learning factory facility at Győr. Procedia Manufacturing, 23, 105-110.

[19] Johannsmeier, L., \& Haddadin, S. (2016). A hierarchical human-robot interactionplanning framework for task allocation in collaborative industrial assembly processes. IEEE Robotics and Automation Letters, 2(1), 41-48.

[20] Cesta, A., Orlandini, A., \& Umbrico, A. (2018). Fostering robust human-robot collaboration through AI task planning. Procedia CIRP, 72, 1045-1050.

[21] Koppenborg, M., Lungfiel, A., Naber, B., \& Nickel, P. (2013). Auswirkung von Autonomie und Geschwindigkeit in der virtuellen Mensch-RoboterKollaboration. Gesellschaft für Arbeitswissenschaft (Hrsg.), Chancen durch Arbeits-, Produkt-und Systemgestaltung, 417-420.

[22] Matheson, E., Minto, R., Zampieri, E. G., Faccio, M., \& Rosati, G. (2019). HumanRobot Collaboration in Manufacturing Applications: A Review. Robotics, 8(4), 100.

[23] Lee, E., Barthelmey, A., Reckelkamm, T., Kang, H., \& Son, J. (2019, October). A Study on Human-Robot Collaboration based Hybrid Assembly System for Flexible Manufacturing. In IECON 2019-45th Annual Conference of the IEEE Industrial Electronics Society (Vol. 1, pp. 4197-4202). IEEE.

[24] Hegenberg, J., Schimpf, D. W., Fischer, N., \& Schmidt, L. (2018). Fallstudie zur Roboterunterstützung des Menschen bei manueller Montage. Zeitschrift für Arbeitswissenschaft, 72(4), 239-251.

[25] Kremer, D., \& Hermann, S. Arbeitsplätze für die Mensch-Roboter-Kollaboration inklusionsförderlich und wirtschaftlich gestalten.

[26] Funk, M., Kosch, T., \& Schmidt, A. (2016, September). Interactive worke assistance: comparing the effects of in-situ projection, head-mounted displays, tablet, and paper instructions. In Proceedings of the 2016 ACM International Joint Conference on Pervasive and Ubiquitous Computing (pp. 934-939).

[27] Schenk, M., Haase, T., Keller, A., \& Berndt, D. (2016). Herausforderungen der Mensch-Technik-Interaktion für die Gestaltung zukünftiger Arbeitssysteme Schlick, C.(Hg.): Megatrend Digitalisierung. Potentiale der Arbeits-und Betriebsorganisation. neue Ausgabe. Berlin: Gito (Schriftenreihe der Wissenschaftliche Gesellschaft für Arbeits-und Betriebsorganisation (WGAB) eV, 1), 131-140.

[28] Zäh, M., Wiesbeck, M., Engstler, F., Friesdorf, F., Schubö, A., Stork, S., ... \& Wallhoff, F. (2007). Kognitive Assistenzsysteme in der manuellen Montage. In wt Werkstattstechnik online (Vol. 97, No. 9, pp. 644-650).

[29] Lupinetti, K., Bonino, B., Giannini, F., \& Monti, M. (2019, June). Exploring the Benefits of the Virtual Reality Technologies for Assembly Retrieval Applications. In International Conference on Augmented Reality, Virtual Reality and Computer Graphics (pp. 43-59). Springer, Cham.

[30] Nilsson, S., \& Johansson, B. (2008). Acceptance of augmented reality instructions in a real work setting. In CHI'08 extended abstracts on Human factors in computing systems (pp. 2025-2032)

[31] Hou, L., Wang, X., \& Truijens, M. (2015). Using augmented reality to facilitate piping assembly: an experiment-based evaluation. Journal of Computing in Civil Engineering, 29(1), 05014007.

[32] Danielsson, O., Syberfeldt, A., Brewster, R., \& Wang, L. (2017). Assessing instructions in augmented reality for human-robot collaborative assembly by using demonstrators. Procedia CIRP, 63, 89-94. 